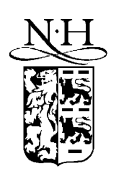

\title{
Noncommutative D-brane and open string in pp-wave background with $B$-field
}

\author{
C.-S. Chu ${ }^{\text {a }}$ P.-M. Ho ${ }^{\text {b }}$ \\ a Centre for Particle Theory, Department of Mathematical Sciences, University of Durham, \\ Durham DH1 3LE, UK \\ b Department of Physics, National Taiwan University, Taipei 106, Taiwan, ROC
}

Received 5 April 2002; accepted 2 May 2002

\begin{abstract}
The open string ending on a D-brane with a constant $B$-field in a pp-wave Ramond-Ramond background is exactly solvable. The theory is controlled by three dimensionful parameters: $\alpha^{\prime}$, the mass parameter (RR background times the lightcone momentum) and the $B$-field. We quantize the open string theory and determine the full noncommutative structure. In particular, we find a fully noncommutative phase space whose noncommutativity depends on all these parameters. The lightcone Hamiltonian is obtained, and as a consequence of the nontrivial commutation relations of the theory, new features of the spectrum are noted. Various scaling limits of the string results are considered. Physical implications are discussed. () 2002 Elsevier Science B.V. All rights reserved.
\end{abstract}

Keywords: Non-commutative geometry; Open string; D-brane; pp-wave

\section{Introduction}

Recently a new maximal supersymmetric IIB supergravity background, the so-called pp-wave RR background, was discovered [1]. The pp-wave background consists of a plane wave metric, supported by a homogeneous RR 5-form flux

$$
\begin{aligned}
& d s^{2}=-f^{2} x^{i} x^{i}\left(d x^{+}\right)^{2}+2 d x^{+} d x^{-}+d x^{i} d x^{i}, \quad i=1, \ldots, 8, \\
& F_{5}=f d x^{+} \wedge\left(d x^{1} \wedge d x^{2} \wedge d x^{3} \wedge d x^{4}+d x^{5} \wedge d x^{6} \wedge d x^{7} \wedge d x^{8}\right) .
\end{aligned}
$$

A constant Euclidean metric $g_{i j}$ can be introduced easily. We will do so in Section 3. This background has 32 supersymmetries and is related to the $A d S_{5} \times S^{5}$ background [1-3] by a

E-mail addresses: chong-sun.chu@durham.ac.uk (C.-S. Chu), pmho@phys.ntu.edu.tw (P.-M. Ho). 
Penrose limit [4]. Moreover, it is remarkable that string theory in this background is exactly solvable [5]. The understanding of the properties of string theory in this background is very valuable and is of great interest. The Green-Schwarz formulation of closed string in ppwave background has been performed by Metsaev [5] (see also [6,7]). Boundary states for the lightcone GS strings in pp-wave background has been constructed [8]. The covariant NSR formulation remains illusive however.

It is natural to ask whether there is a meaning of the "Penrose limit" for the celebrated AdS/CFT correspondence [9]. Based on the spectrum of the lightcone Hamiltonian, Berenstein, Maldacena and Nastase [10] had put forward a remarkable proposal that string theory on this pp-wave background is dual to the large $N$ limit of a certain subsector of the 4-dimensional supersymmetric $\mathcal{N}=4 S U(N)$ gauge theory. Various aspects as well as generalizations had been considered in [11-21].

D-branes are basic objects in the nonperturbative formulation of string theory. One of the developments in the last few years of string theory is the realization that a constant $B$-field on a D-brane leads to noncommutative geometry on the D-brane worldvolume [22-25]. In this setting, the $B$-field and the string length square $\alpha^{\prime}$ are both dimensionful. Therefore, it is conceivable that a double scaling limit may be taken so that certain dimensionful parameter remains in the low energy limit $\alpha^{\prime} \rightarrow 0$. This is precisely what Seiberg and Witten did [25] and remarkably they obtained as the low energy limit a noncommutative field theory with the dimensionful noncommutativity parameters $\theta^{\mu \nu}$. It is an interesting question whether there are other string backgrounds for which new kind of noncommutative geometries arise.

Now string in pp-wave is exactly solvable (at least in the GS formulation), and there is a mass parameter $m$ (see (3.2) below) in addition to $\alpha^{\prime}$. If we also turn on a $B$-field, then we have three dimensionful parameters at our disposal. It is therefore very natural to consider a D-brane with a constant $B$-field sitting in the pp-wave background and check whether any new kind of quantum geometry arises, and to consider the possible scaling limits in the low energy. This is the main motivation of our work.

We recall that from the point of view of [24,26], noncommutative geometry on the Dbrane worldvolume is a direct consequence of the open string mixed boundary condition that occurs due to the nonzero $B$-field. This gives rise to a noncommutativity of the string zero mode $x_{0}^{i}$ among themselves, but leaves the momentum zero mode $p_{0}^{i}$ commuting among themselves. As it turns out, we will find that by turning on a mass parameter $m, p_{0}^{i}$ also becomes noncommuting. Thus the phase space of the zero modes $x_{0}, p_{0}$ becomes fully noncommuting, see (3.27)-(3.29). Moreover, we find that the noncommutativity depends on the lightcone momentum $p^{+}$. This is one of the main results of this paper. Its physical meaning is intriguing and further understanding of it will be important. An immediate consequence of the commutation relations we obtain is that the spectrum of the lightcone Hamiltonian is modified in a nontrivial manner by the $B$-field (see (3.40)). These are possible since $p^{+}$is central in the pp-wave supersymmetry algebra.

The paper is organized as follows. In Section 2, we first present a D-brane configuration that preserves half of the 32 supersymmetries. We show that it is allowed to put a constant $B$-field on its worldvolume. Section 3 is devoted to the quantization of the open string ending on this D-brane. In Section 3.1, we construct the complete mode expansion of an open string ending on a D-brane with $B$-field in the pp-wave background. The zero mode 
part has a nontrivial dependence on the worldsheet $\sigma$-coordinate (see (3.15)) and is more complicated than the case without $B$-field. Also we find that the zero mode frequency is affected by a constant background $B$-field, but not for the higher oscillation modes. In Section 3.2, we review the procedure for determining the commutation relations of the theory. The starting point is the symplectic form (3.22). We explain why it is consistent and use it to derive the basic commutation relations (3.27)-(3.30) for the string modes. As a result of these commutation relations, the boundary commutators of the string coordinates and string canonical momentum are modified, and only at the boundary, as in (3.45)(3.47). The spectrum of the lightcone Hamiltonian is obtained. As a consequence of these commutation relations, we find that the spectrum of the nonzero modes depend on the mode number $n$ in a more complicated manner; we also noted an interesting splitting in the zero mode spectrum, see (3.40). In Section 3.3, we carry out the constrained quantization of Dirac and obtain the same result. In Section 4, we discuss various scaling limits and discuss their physical meanings. We conclude the paper with further discussions.

\section{BPS D-brane with $B$-field in pp-wave}

Consider a flat D-brane in the pp-wave background, with a constant $B$-field turned on in its worldvolume. This is a BPS configuration preserving half of the $32 \mathrm{pp}$-wave supersymmetries. This can be easily seen from the limiting procedure considered in [2, 3 ] that relate brane probe solution in $A d S \times S$ to brane probe solution in the Penroselimited pp-wave or Minkowskian background. In this section, we will use the $\kappa$-symmetric formulation of $\mathrm{D} p$-brane (see, for example, [27-29]) to show this explicitly. It will also allow us to see how the D-brane is stuck at the origin from the point of view of the BornInfeld theory, see (2.21).

Given a D-brane probe, the surviving supersymmetries satisfies the condition

$$
(1-\Gamma) \xi=0
$$

where $\Gamma$ is a projector that depends on the details of the brane configuration. The explicit form of $\Gamma$ was obtained, for example, in [30], where it was shown that the Born-Infeld field strength amounts to a relative rotation of the left and right moving fields. In order to be self contained, we recall that $\Gamma$ is given by

$$
\Gamma=e^{-a / 2} \Gamma_{(0)}^{\prime} e^{a / 2},
$$

where $a$ is a matrix given below and

$$
\Gamma_{(0)}^{\prime}= \begin{cases}\left(\Gamma_{11}\right)^{\frac{p-2}{2}} \Gamma_{(0)} & \text { IIA } \\ \left(\sigma_{3}\right)^{\frac{p-3}{2}} i \sigma_{2} \otimes \Gamma_{(0)} & \text { IIB }\end{cases}
$$

and

$$
\Gamma_{(0)}=\frac{1}{(p+1) ! \sqrt{-\operatorname{det} G}} \epsilon^{i_{1} \cdots i_{p+1}} \partial_{i_{1}} X^{M_{1}} \cdots \partial_{i_{p+1}} X^{M_{p+1}} \Gamma_{M_{1} \cdots M_{p+1}}^{\prime} .
$$


As usual, the matrix $\Gamma_{M_{1} \cdots M_{p+1}}^{\prime}$ is the antisymmetrized product of the $\Gamma_{M_{k}}^{\prime}$ with the $\Gamma_{M}^{\prime}$ being the 10-dimensional $\Gamma$-matrices in the coordinate basis defined by

$$
\Gamma_{M}^{\prime}:=E_{M}^{A} \Gamma_{A},
$$

where the $\Gamma_{A}$ are flat space $\Gamma$-matrices. The metric $G_{i j}$ is the induced worldvolume metric

$$
G_{i j}=\partial_{i} X^{M} \partial_{j} X^{N} g_{M N}
$$

To define the matrix $a$ appearing in (2.2) we need to introduce the modified 2-form field strength $\mathcal{F}$ which is related to the Born-Infeld field strength $F=d A$ by

$$
\mathcal{F}=F-\underline{B},
$$

where $\underline{B}$ is the pull-back of the target space NS-NS 2-form potential to the worldvolume. The matrix $a$ depends only on the worldvolume Born-Infeld field strength and is given by

$$
a= \begin{cases}-\frac{1}{2} Y_{j k} \gamma^{j k} \Gamma_{11} & \text { IIA, } \\ \frac{1}{2} Y_{j k} \sigma_{3} \otimes \gamma^{j k} & \text { IIB, }\end{cases}
$$

the $\gamma^{j k}$ being worldvolume $\gamma$ matrices,

$$
\gamma_{i}=\partial_{i} X^{M} \Gamma_{M}^{\prime}
$$

and $Y$ is a function of $\mathcal{F}$. The relation in the frame basis of the worldvolume (hatted indices) is

$$
Y_{\hat{j} \hat{k}}:=\tan ^{-1} \mathcal{F}_{\hat{j} \hat{k}}
$$

Specifically, let us consider a $\mathrm{D} p$-brane spanning the directions $\left(+,-, i_{2}, \ldots, i_{p}\right)$, where $X^{ \pm}=\left(X^{0} \pm X^{9}\right) / \sqrt{2}$ and $\left(i_{2}, \ldots, i_{p}\right) \in(1, \ldots, 8)$. Denote the worldvolume coordinates of the D-brane by $\left(\tau, \xi^{i}\right), i=1, \ldots, p$. In the lightcone gauge,

$$
X^{+}(\tau, \xi)=p^{+} \tau
$$

We also take the physical gauge

$$
X^{-}(\tau, \xi)=\xi^{1}, \quad X^{i_{k}}(\tau, \xi)=\xi^{i_{k}}, \quad k=2, \ldots, p .
$$

The transverse directions $X^{a}(\tau, \xi)$, then describe the embedding of the D-brane.

We will consider a constant $B$-field with nonvanishing components $B_{i j}, i, j \in$ $(1, \ldots, 8)$. The pull-back $B$-field is the same as the target space components, and therefore we will not distinguish them anymore. As for the pull-back metric, we have

$$
G_{\mu \nu}=G_{\mu \nu}^{(0)}+\partial_{\mu} X^{a} \partial_{\nu} X^{a}
$$

where $G_{\mu \nu}^{(0)}$ is the metric

$$
G^{(0)}=\left(\begin{array}{ccc}
-u^{2} & p^{+} & \\
p^{+} & 0 & \\
& & \mathbb{1}_{(p-1) \times(p-1)}
\end{array}\right), \quad u^{2}:=p^{+2} f^{2}\left(\sum_{i=2}^{p} \xi^{i 2}+X^{a 2}\right) .
$$


Now, it is easy to see that for a flat D-brane described by

$$
X^{a}(\tau, \xi)=\text { const, }
$$

we have

$$
\Gamma_{(0)}=\Gamma_{+,-, i_{2}, \ldots, i_{p}} .
$$

This is independent of $B$ and $m$ and the D-brane preserves half supersymmetries. Using the form of the Killing spinor given in [1] and the fact that the brane is stuck at the origin (see (2.21) below), it is easy to check that $\Gamma_{(0)}^{\prime}$ preserve half of the supersymmetry for $p=3,5,7$. This agrees with the open string analysis of [31]. ${ }^{1}$ Branes at angle [31] can be discussed similarly.

Finally we check that the brane configuration satisfies the equations of motion. In our case, since we are using the physical gauge, the equations of motion are derived from the gauge fixed form of the Dirac-Born-Infeld action, including the WZ-term,

$$
I=\int d^{p+1} \xi \sqrt{-\operatorname{det}(G+\mathcal{F})}+\int \underline{C}:=I_{\mathrm{DBI}}+I_{\mathrm{WZ}}
$$

where $G_{\mu \nu}$ is the induced worldvolume metric (2.13) in the physical gauge and $\underline{C}$ is the pullback to the worldvolume of the RR 4-form potential. The inclusion of the WZ term is crucial in checking that the equation of motion is satisfied for brane configurations with nontrivial embeddings [32]. However, for the trivial embedding (2.15), the WZ term does not play any role. The equation of motion reads

$$
\begin{aligned}
& \Delta^{-1} \partial_{\mu}\left(\Delta\left\{(G+\mathcal{F})^{-1}+(G-\mathcal{F})^{-1}\right\}^{\mu \nu} \partial_{\nu} X^{a}\right)-m^{2} X^{a}=0, \\
& \partial_{\mu}\left(\Delta\left\{(G+\mathcal{F})^{-1}-(G-\mathcal{F})^{-1}\right\}^{\mu \nu}\right)=0,
\end{aligned}
$$

where

$$
\Delta:=\sqrt{-\operatorname{det}(G+\mathcal{F})}
$$

The only constant solution of Eq. (2.18) is

$$
X^{a}=0 .
$$

The absence of flat directions is because they are lifted by the potential well created by the mass term. Eq. (2.21) implies that

$$
u^{2}=p^{+2} f^{2} \sum_{i=2}^{p} \xi^{i 2} \text {. }
$$

Note that it is independent of neither $\tau$ nor $\xi^{1}$. It is then easy to check that

$$
\mathcal{F}_{i j}=\text { const }
$$

is a solution to (2.19). Hence our claim is justified. In the following, we will turn to the open string description for these D-branes. For simplicity, we will take the gauge $\mathcal{F}=B$, where $A=0$.

\footnotetext{
1 We thank Atish Dabholkar, Jaume Gomio and Shahrokh Parvizi for useful e-mail exchanges.
} 


\section{Noncommutative D-brane with $B$-field in pp-wave}

The main objective of this paper is to work out the effects of $B$-field on the quantization of the open string theory ending on a D-brane in the pp-wave background. We will carry out this analysis in the GS formulation.

\subsection{Open string mode expansion}

Recall that for a flat D-brane in Minkowskian spacetime, the open string GS action was obtained from lightcone gauge fixing the covariant action [33], in which the latter can be obtained by substituting in the superfield background that solves the IIB supergravity constraints. With a constant $B_{\mu \nu}$ field turned on, it induces new nonzero components in the superfields $B_{\mu \alpha}$ and $B_{\alpha \beta}$. However, these terms all cancel themselves out in the action and the only effect is the addition of the usual bosonic $B$-field coupling to the target space [34]. A coupling to the fermionic spacetime variables would arise only if $B$ were not closed. It was also shown that one can use the usual (i.e., the one for $B=0$ ) lightcone gauge fixing condition so long as $B_{0 \mu}=B_{9 \mu}=0$ [34]. This is exactly the setting we considered in Section 2.

The $\kappa$-symmetric formulation of closed string in pp-wave background was analysed in [5]. In the lightcone gauge, the theory is exactly solvable and consists of eight massive bosons and fermions. Turing on the $B$-field, and carrying out a similar analysis as in [5, 34], one finds that the open string has the bosonic action in the lightcone gauge,

$$
S=\frac{1}{4 \pi \alpha^{\prime}} \int d^{2} \sigma\left[g_{i j}\left(\eta^{\alpha \beta} \partial_{\alpha} X^{i} \partial_{\beta} X^{j}+m^{2} X^{i} X^{j}\right)+\epsilon^{\alpha \beta} \partial_{\alpha} X^{i} \partial_{\beta} X^{j} B_{i j}\right],
$$

where $i, j=1, \ldots, p$, and $B$ is turned on only in the directions $2, \ldots, p$. Following [7], we have introduced the parameter

$$
m:=\alpha^{\prime} p^{+} f
$$

and we have taken the length of the $\sigma$-interval to be $\pi$; therefore, strings with different lightcone momentum have different $m$. We ignored the transverse coordinates in (3.1) since the quantization of them is standard. The fermionic sector will not affect the result and can be considered separately. We have introduced the Euclidean metric $g_{i j}$ for the sake of the scaling limits to be considered in the next section. Note that in our normalization, $B, m$ are dimensionless. The equation of motion is

$$
\left(-\partial_{\tau}^{2}+\partial_{\sigma}^{2}-m^{2}\right) X^{i}=0
$$

and the boundary condition is

$$
\partial_{\sigma} X^{i}+\partial_{\tau} X^{j} B_{j}{ }^{i}=0
$$

at $\sigma=0, \pi$. Indices $i, j$ are raised and lowered by $g_{i j}$. The lightcone Hamiltonian is $[5,6]$

$$
H=\frac{1}{4 \pi \alpha^{\prime} p^{+}} \int_{0}^{\pi} d \sigma\left(\left(\partial_{\tau} X\right)^{2}+\left(\partial_{\sigma} X\right)^{2}+m^{2} X^{2}\right),
$$


We are interested in the effects of the constraint (3.4) to the quantization of the theory. The situation is exactly the same as in [24]. The boundary condition (3.4) implies that

$$
\begin{aligned}
& 2 \pi \alpha^{\prime}\left[P^{k}(\tau, 0), P^{j}\left(\tau, \sigma^{\prime}\right)\right] B_{k}{ }^{i}=-\partial_{\sigma}\left[X^{k}(\tau, \sigma), P^{j}\left(\tau, \sigma^{\prime}\right)\right]_{\sigma=0} M_{k}{ }^{i}, \\
& 2 \pi \alpha^{\prime}\left[P^{k}(\tau, 0), X^{j}\left(\tau, \sigma^{\prime}\right)\right] B_{k}{ }^{i}=-\partial_{\sigma}\left[X^{k}(\tau, \sigma), X^{j}\left(\tau, \sigma^{\prime}\right)\right]_{\sigma=0} M_{k}{ }^{i},
\end{aligned}
$$

where $P^{k}$ is the conjugate momentum

$$
2 \pi \alpha^{\prime} P^{k}(\tau, \sigma)=\partial_{\tau} X^{k}+\partial_{\sigma} X^{j} B_{j}{ }^{k},
$$

and

$$
M_{k}^{i}=\delta_{k}^{i}-B_{k}^{j} B_{j}^{i} .
$$

These simple relations show that the standard canonical commutation relations for $B=0$ [5] are no longer valid when $B \neq 0$.

Without loss of generality, we will assume the metric is already in the diagonal form

$$
g_{i j}=\lambda \delta_{i j} \text {. }
$$

In addition we will consider the case in which the $B$-field takes the form

$$
B_{i j}=\left(\begin{array}{cc}
0 & B \\
-B & 0
\end{array}\right)
$$

and focus on $X^{2}, X^{3}$. We remark that the action (3.1) is $S O(2)$-invariant, and so the angular momentum is conserved. The mode expansion takes the form

$$
X^{i}=X_{(0)}^{i}+X_{(1)}^{i},
$$

where $^{2}$

$$
\begin{aligned}
X_{(0)}^{i}= & \left(x_{0}^{i} \cos \omega_{0} \tau+2 \alpha^{\prime} p_{0}^{i} \frac{\sin \omega_{0} \tau}{\omega_{0}}\right) \cosh \omega_{0} \bar{B} \sigma \\
& +\left(-2 \alpha^{\prime} p_{0}^{j} \cos \omega_{0} \tau+x_{0}^{j} \omega_{0} \sin \omega_{0} \tau\right) B_{j}{ }^{i} \frac{\sinh \omega_{0} \bar{B} \sigma}{\omega_{0} \bar{B}}
\end{aligned}
$$

is the "zero mode" part, and

$$
X_{(1)}^{k}=\sqrt{2 \alpha^{\prime}} \sum_{n \neq 0} e^{-i \omega_{n} \tau}\left(i \frac{\alpha_{n}^{k}}{\omega_{n}} \cos n \sigma-\frac{\alpha_{n}^{j}}{n} B_{j}{ }^{k} \sin n \sigma\right),
$$

\footnotetext{
${ }^{2}$ It is straightforward to extend the two-dimensional case to the generic case. Assuming that $B_{\mu \nu}$ is invertible, we can always decompose it as $U^{T} b U$ by an orthogonal transformation $U$, where $b$ is a matrix of diagonal blocks of the form (3.11). Then the zero mode solution is

$$
X_{(0)}^{i}=x_{+}^{j}\left(U^{T} \exp \left(+\left(a b^{-1} \tau-a \sigma\right)\right) U\right)_{j}{ }^{i}+x_{-}^{j}\left(U^{T} \exp \left(-\left(a b^{-1} \tau-a \sigma\right)\right) U\right)_{j}{ }^{i},
$$

where the matrix $a$ is a diagonal matrix defined by

$$
a^{2}=\frac{m^{2}}{1-b^{-2}} .
$$

Note that $a$ is well defined by this relation because $b^{-2}$ is diagonal with negative entries.
} 
is the nonzero mode part. The constant $\bar{B}$

$$
\bar{B}:=B / \lambda,
$$

is the eigenvalue of the matrix $B_{i}{ }^{j}$; and the frequencies are defined by

$$
\omega_{0}:=\frac{m}{\sqrt{1+\bar{B}^{2}}}>0, \quad \text { and } \quad \omega_{n}:=\operatorname{sign}(n) \sqrt{n^{2}+m^{2}}, n \neq 0 .
$$

Several remarks about the "zero modes" are in order.

1. By "zero modes" here, we simply refer to the modes with the lowest frequency. We stress that the zero modes are very different in structure compared to the oscillation modes. One important difference between the zero mode part and the oscillation part is that the zero mode part $X_{(0)}^{i}$ actually satisfies (3.3) and (3.4) for all $0 \leqslant \sigma \leqslant \pi$. This is the same as in the flat case $(m=0)$ [24].

2. Note that the zero mode structure is more complicated than the $B=0$ case, where $X_{(0)}^{i}$ was simply independent of $\sigma$ [5].

3. In our expansion (3.15), the coefficients $x_{0}$ and $p_{0}$ are identified conveniently such that they have the correct dimensions. It is a simple choice and it is possible to rescale them by dimensionless factors.

4. Finally, we note that naively one may have expected that, since turning on a constant $B$-field has no effect on the equation of motion (propagation), the zero mode will have the frequency $m$ as in the $B=0$ case [5]. However, it is easy to check that when $B \neq 0$, this frequency does not give rise to any solution that can satisfy both the equation of motion and the boundary condition. The modification of the zero mode frequency by the external $B$-field through the boundary condition is an interesting effect. We recall that in the flat case for a neutral string, turning on a $B$-field does not change the mode frequency. In the case of a charged string [35], all the frequencies are modified by the background field. Our case here is intermediate, turning on a $B$-field only modify the zero mode frequency. This is another difference between the zero modes and the oscillation modes.

The canonical momentum also splits into a sum

$$
P^{k}=P_{(0)}^{k}+P_{(1)}^{k}
$$

of the zero mode part

$$
\begin{aligned}
2 \pi \alpha^{\prime} P_{(0)}^{k}= & \left(-x_{0}^{j} \omega_{0} \sin \omega_{0} \tau+2 \alpha^{\prime} p_{0}^{j} \cos \omega_{0} \tau\right) M_{j}{ }^{k} \cosh \omega_{0} \bar{B} \sigma \\
& +\left(2 \alpha^{\prime} p_{0}^{j} \sin \omega_{0} \tau+x_{0}^{j} \omega_{0} \cos \omega_{0} \tau\right)\left(B g^{-1} M\right)_{j}{ }^{k} \frac{\sinh \omega_{0} \bar{B} \sigma}{\bar{B}},
\end{aligned}
$$

and the nonzero mode part

$$
2 \pi \alpha^{\prime} P_{(1)}^{k}=\sqrt{2 \alpha^{\prime}} \sum_{n \neq 0} e^{-i \omega_{n} \tau}\left(\alpha_{n}^{j} M_{j}{ }^{k} \cos n \sigma+i \frac{m^{2}}{n \omega_{n}} \alpha_{n}^{j} B_{j}{ }^{k} \sin n \sigma\right) .
$$

Note that (3.15), (3.16), (3.21) and (3.20) reduce smoothly to the usual flat space expressions in the limit $m \rightarrow 0$. 


\subsection{Open string quantization in pp-wave with B-field}

To quantize the theory we will follow the procedure adopted in [24,26], where interpretations in terms of noncommutative geometry were emphasized. This method is equivalent to the canonical quantization performed in [35]. That paper also gave the first instance demonstrating how noncommutativity of the zero modes can arise in the presence of a background field. Its physical implications had been studied in $[35,36]$.

First we need the symplectic form

$$
\Omega=\int_{0}^{\pi} d \sigma g_{i j} \mathbf{d} P^{i} \mathbf{d} X^{j} .
$$

That this is the correct symplectic form of the theory can be justified from an analysis of the constraint structure of the theory. Without going into the details, one can already see this since the constraint (3.4) is imposed only at the boundary. It is clear that the constrained quantization method will give a Dirac bracket which is modified and is possibly different from the original Poisson bracket only at the boundary. (We will present the constrained quantization in the next subsection.) This is a measure zero set and so the symplectic form takes the standard form (3.22).

Of course, this does not mean that the commutation relations of the theory are unmodified. This is felt through the modified form of the mode expansion (3.15), (3.16), (3.20) and (3.21). By substituting in the mode expansions (3.12) and (3.19) and evaluate the $\sigma$ integral, the symplectic form (3.22) of the theory can be thought of as the symplectic form defined for the string modes. This procedure is consistent since, using (3.3) and (3.4), it is easy to check that (3.22) is independent of $\tau$ [37]. As a result, one can take the resulting expression as the symplectic form for the string modes. The commutation relation of the string modes can be then obtained from (3.22) by inverting the symplectic matrix.

Explicitly we obtain the following symplectic form for the string modes

$$
\begin{aligned}
2 \pi \alpha^{\prime} \Omega= & 2 \alpha^{\prime} \tilde{c} M_{i j} \mathbf{d} p_{0}^{i} \mathbf{d} x_{0}^{j}-\frac{\omega_{0} c}{2 \bar{B}}\left(M g^{-1} B\right)_{i j} \mathbf{d} x_{0}^{i} \mathbf{d} x_{0}^{j} \\
& -\left(2 \alpha^{\prime}\right)^{2} \frac{c}{2 \omega_{0} \bar{B}}\left(M g^{-1} B\right)_{i j} \mathbf{d} p_{0}^{i} \mathbf{d} p_{0}^{j}+i 2 \pi \alpha^{\prime} \sum_{n=1}^{\infty} \frac{M_{i j}^{n}}{\omega_{n}} \mathbf{d} \alpha_{n}^{i} \mathbf{d} \alpha_{-n}^{j},
\end{aligned}
$$

where the "metric" $M_{i j}^{n}$ is defined by

$$
M_{i j}^{n}:=g_{i j}-\frac{\omega_{n}^{2}}{n^{2}}\left(B g^{-1} B\right)_{i j} .
$$

Note that $M_{i j}^{n}=M_{i j}$ only when $m=0$. The constants $c, \tilde{c}$ are defined by

$$
\begin{aligned}
& c=\frac{\cosh \left(2 \pi \omega_{0} \bar{B}\right)-1}{2 \omega_{0} \bar{B}} \approx \pi^{2} \omega_{0} \bar{B}+\cdots, \\
& \tilde{c}=\frac{\sinh \left(2 \pi \omega_{0} \bar{B}\right)}{2 \omega_{0} \bar{B}} \approx \pi+\cdots
\end{aligned}
$$


The $\approx$ above gives the leading order expansion in the small $m$ limit. Following [25]. we have been careful in keeping the metric dependence to facilitate the discussion of scaling limits. This will be done in the next section.

Inverting the symplectic matrix, we obtain the following commutation relations for the zero modes and for the oscillation modes

$$
\begin{aligned}
& {\left[x_{0}^{i}, p_{0}^{j}\right]=i\left(M^{-1}\right)^{i j} \frac{\pi \omega_{0} \bar{B}}{\tanh \pi \omega_{0} \bar{B}},} \\
& {\left[x_{0}^{i}, x_{0}^{j}\right]=i 2 \pi \alpha^{\prime}\left(g^{-1} B M^{-1}\right)^{i j},} \\
& {\left[p_{0}^{i}, p_{0}^{j}\right]=i \frac{\pi \omega_{0}^{2}}{2 \alpha^{\prime}}\left(g^{-1} B M^{-1}\right)^{i j},} \\
& {\left[\alpha_{n}^{i}, \alpha_{s}^{j}\right]=\omega_{n} M_{n}^{i j} \delta_{n+s},}
\end{aligned}
$$

where

$$
M_{n}^{i j}=\left(\frac{1}{g+\frac{\omega_{n}}{n} B} g \frac{1}{g-\frac{\omega_{n}}{n} B}\right)^{i j}
$$

is the inverse of $M_{i j}^{n}$ for each fixed $n$. It represents a metric that is mode-dependent. This is in contrast with the flat case, where it was found that [24] the oscillator's commutation relations are determined by the same open string metric $M^{i j}$ as the zero modes. Here we find that each level of the oscillators see a different metric. A consequence of this is the more complicated $n$-dependence in the spectrum of the Hamiltonian (3.40). It may be interesting to investigate to what extent one can think of $M_{n}^{i j}$ as a mode-dependent openstring metric. Note that in the form (3.10) of the metric,

$$
M_{n}^{i j}=g^{i j}\left(1+\frac{\omega_{n}^{2}}{n^{2}} \bar{B}^{2}\right)^{-1}
$$

is diagonal and is a monotonic increasing function of $n$ which is bounded above by $M^{i j}$. Note also that by turning on the mass parameter $m, p_{0}^{i}$ also become noncommuting themselves; and the phase space of $x_{0}, p_{0}$ becomes fully noncommuting. The noncommutative parameters depends on the background of the pp-wave, and on the lightcone momentum $p^{+}$.

In the above, we have considered a single string with a single $p^{+}$. If one wants to perform calculations with many string states, then it will be useful to introduce a basis of oscillators whose commutation relations are independent of $p^{+}$. For the nonzero modes, we can introduce the following basis of oscillators

$$
a_{n}^{i}:=\sqrt{\frac{1+\frac{\omega_{n}^{2} \bar{B}^{2}}{n^{2}}}{\omega_{n} / \lambda}} \alpha_{-n}^{i}, \quad \bar{a}_{n}^{i}:=\sqrt{\frac{1+\frac{\omega_{n}^{2}}{n^{2}} \bar{B}^{2}}{\omega_{n} / \lambda}} \alpha_{n}^{i}, \quad n>0 .
$$

They obey the relations

$$
\left[\bar{a}_{n}^{i}, a_{s}^{j}\right]=\delta^{i j} \delta_{n s}, \quad\left[a_{n}^{i}, a_{s}^{j}\right]=0 .
$$


Note the $B$-dependence in the rescaling. For the zero mode, one may define

$$
a_{0}^{i}:=\frac{w}{2}\left(x_{0}^{i}-i \frac{2 \alpha^{\prime} p_{0}^{i}}{\omega_{0}}\right), \quad \bar{a}_{0}^{i}:=\left(a_{0}^{i}\right)^{\dagger}, \quad \text { with } \quad w:=\sqrt{\frac{\lambda m^{2}}{\alpha^{\prime} \omega_{0}} \frac{\tanh \pi \bar{B} \omega_{0}}{\pi \bar{B} \omega_{0}},}
$$

in terms of which the commutation relations (3.27)-(3.29) take the simple form of a deformed oscillator algebra

$$
\left[\bar{a}_{0}^{i}, a_{0}^{j}\right]=\delta^{i j}+i \epsilon^{i j} \tanh \pi \bar{B} \omega_{0}, \quad\left[a_{0}^{i}, a_{0}^{j}\right]=0,
$$

which is $S O(2)$-invariant. Or one may introduce

$$
a_{ \pm}:=\frac{a_{0}^{1} \pm i a_{0}^{2}}{\sqrt{2\left(1 \mp \tanh \pi \bar{B} \omega_{0}\right)}}, \quad \bar{a}_{ \pm}:=\left(a_{ \pm}\right)^{\dagger},
$$

which obey the $U(1)$ invariant commutation relations

$$
\left[\bar{a}_{A}, a_{B}\right]=\delta_{A B}, \quad\left[a_{A}, a_{B}\right]=0, \quad A, B=+,- \text {. }
$$

In terms of them, the zero mode part of the string coordinates can be written as, for example,

$$
X_{(0)}^{i}=\frac{e^{i \omega_{0} \tau}}{w}\left(a_{0}^{i} \cosh \omega_{0} \bar{B} \sigma-i a_{0}^{j} B_{j}{ }^{i} \frac{\sinh \omega \bar{B} \sigma}{\bar{B}}\right)+\text { h.c. }
$$

The lightcone Hamiltonian (3.5) takes the diagonal form

$$
\begin{aligned}
H= & \frac{\omega_{0} \cosh ^{2} \pi \bar{B} \omega_{0}}{p^{+}}\left(\left(1-\tanh \pi \bar{B} \omega_{0}\right) a_{+} \bar{a}_{+}+\left(1+\tanh \pi \bar{B} \omega_{0}\right) a_{-} \bar{a}_{-}+1\right) \\
& +\frac{1}{p^{+}} \sum_{n=1}^{\infty} \frac{1+\bar{B}^{2}}{1+\frac{\omega_{n}^{2} \bar{B}^{2}}{n^{2}}} \omega_{n} a_{n}^{i} \bar{a}_{n}^{i},
\end{aligned}
$$

where we have written the Hamiltonian in a normal ordered form, and have dropped the infinite additive constant arising from the nonzero modes. This will be cancelled with the fermionic oscillators [7]. The factor 1 in (3.40) came from the normal ordering of the zero mode oscillator $a_{ \pm}$and remember that we are considering two string coordinates $X^{2,3}$.

The Fock vacuum of the theory is defined by

$$
\bar{a}_{ \pm}|0\rangle=0, \quad \bar{a}_{n}^{i}|0\rangle=0, n>0 .
$$

It is $S O(2)$-invariant. In choosing the definition of the vacuum, we have been guided by the requirement of $S O(2)$ invariance of the theory. Turning on a $B$-field in the 2-3 directions preserve this symmetry. Note that the spectrum for the nonzero modes depend on $n$ in a more complicated way due to $B \neq 0$. Note also that the degeneracy of the states $a_{ \pm}|0\rangle$ is lifted when $B \neq 0$. We expect that this splitting of the string spectrum to have interesting physical consequences.

Finally we derive the intrinsic commutation relations of the theory in terms of $X^{i}$ and $P^{i}$. Using the relations above, one can easily obtain

$$
\left[X^{i}(\tau, \sigma), X^{j}\left(\tau, \sigma^{\prime}\right)\right]=i 2 \pi \alpha^{\prime} B^{i j} f\left(\sigma+\sigma^{\prime}\right),
$$


where the function $f(\sigma)$ is defined on $\sigma \in[0,2 \pi]$ as

$$
f(\sigma):=\frac{1}{c}\left[c \cosh \left(\omega_{0} \bar{B} \sigma\right)-\tilde{c} \sinh \left(\omega_{0} \bar{B} \sigma\right)\right]-\frac{2}{\pi} \sum_{n=1}^{\infty} \frac{1}{n}\left(1+\frac{\omega_{0}^{2} \bar{B}^{2}}{n^{2}}\right)^{-1} \sin n \sigma .
$$

It is easy to show that

$$
f(\sigma)=\left(1+\bar{B}^{2}\right)^{-1} \begin{cases}+1, & \sigma=0 \\ -1, & \sigma=2 \pi \\ 0, & 0<\sigma<2 \pi\end{cases}
$$

Thus the commutation relation of $X$ is

$$
\left[X^{i}(\tau, \sigma), X^{j}\left(\tau, \sigma^{\prime}\right)\right]=i 2 \pi \alpha^{\prime}\left(g^{-1} B M^{-1}\right)^{i j} \begin{cases}1, & \sigma=\sigma^{\prime}=0 \\ -1, & \sigma=\sigma^{\prime}=\pi \\ 0, & \text { otherwise }\end{cases}
$$

and is modified only at the endpoints of the open string. The commutator for $P$ themselves can be similarly computed and we obtain

$$
\left[P^{i}(\tau, \sigma), P^{j}\left(\tau, \sigma^{\prime}\right)\right]=i \frac{m^{2}}{2 \pi \alpha^{\prime}}\left(1+\bar{B}^{2}\right) B^{i j} f\left(\sigma+\sigma^{\prime}\right) .
$$

Thus both commutators are given in terms of the same function $f$. As a result, we find

$$
\left[P^{i}(\tau, \sigma), P^{j}\left(\tau, \sigma^{\prime}\right)\right]=i \frac{m^{2}}{2 \pi \alpha^{\prime}} B^{i j} \begin{cases}+1, & \sigma=\sigma^{\prime}=0 \\ -1, & \sigma=\sigma^{\prime}=\pi \\ 0, & \text { otherwise. }\end{cases}
$$

It is intriguing that the commutation relations for the endpoint momentum are nontrivial. Note also it dependent on the parameter $m=\alpha^{\prime} p^{+} f$ in a nontrivial way. This is a new feature of the pp-wave background: the noncommutative space felt by a string depends on its light-cone momentum. Finally we obtain

$$
\left[X^{i}(\tau, \sigma), P^{j}\left(\tau, \sigma^{\prime}\right)\right]=i \frac{g^{i j}}{\pi}\left(1+\sum_{n \neq 0} \cos n \sigma \cos n \sigma^{\prime}\right) .
$$

Note that (3.45) and (3.47) take exactly the same form as in the flat case [24] and is unmodified by $m$. Also note that, unlike the relations for the zero modes, the relations (3.45)-(3.47) are intrinsic and is independent of how the zero modes are identified, nor how the vacuum is chosen.

\subsection{Constrained quantization}

In this subsection, we present the construction of the Dirac bracket following the procedure in [26]. To start with, the standard Poisson bracket is [5]

$$
\begin{aligned}
& \left(X^{i}(\sigma), P_{j}\left(\sigma^{\prime}\right)\right)=\delta_{j}^{i} \delta\left(\sigma, \sigma^{\prime}\right), \quad\left(P_{i}(\sigma), P_{j}\left(\sigma^{\prime}\right)\right)=0, \\
& \left(X^{i}(\sigma), X^{j}\left(\sigma^{\prime}\right)\right)=0 .
\end{aligned}
$$


As first noted in [24], the boundary condition can be treated as a constraint on the phase space

$$
\Phi^{i}(0)=\Phi^{i}(\pi)=0,
$$

where we have introduced

$$
\Phi^{i}(\sigma):=2 \pi \alpha^{\prime} P^{j} B_{j}{ }^{i}+\partial_{\sigma} X^{j} M_{j}{ }^{i} .
$$

Using the lightcone Hamiltonian (3.5) one determines the complete set of second class constraints

$$
\partial_{\sigma}^{2 \mathrm{n}} \Phi^{i}(\sigma)=0, \quad \partial_{\sigma}^{2 \mathrm{n}} \Psi^{i}(\sigma)=0, \quad \mathrm{n}=0,1, \ldots,
$$

where

$$
\Psi^{i}(\sigma):=\partial_{\sigma} P^{i}-\frac{m^{2}}{2 \pi \alpha^{\prime}} X^{j} B_{j}{ }^{i} .
$$

We will denote them by $\phi^{(\alpha k \mathrm{n})}, \alpha=1,2 ; k=2, \ldots, p ; \mathrm{n}=0,1, \ldots$,

$$
\phi^{(1 k \mathrm{n})}=\partial_{\sigma}^{2 \mathrm{n}} \Phi^{k}, \quad \phi^{(2 k \mathrm{n})}=\partial_{\sigma}^{2 \mathrm{n}} \Psi^{k} .
$$

These constraints are consistent with the explicit mode expansion of the fields $X^{i}$ and $P^{i}$ given above.

The Poisson matrix $C^{(\alpha k \mathrm{n})(\beta l \mathrm{~m})}$ of the constraints can be computed easily. The basic ones are the $C^{(\alpha k 0)(\beta l 0)}$ :

$$
\begin{aligned}
& \left(\Phi^{k}(\sigma), \Phi^{l}\left(\sigma^{\prime}\right)\right)=2 \pi \alpha^{\prime}(B M)^{k l}\left[\partial_{\sigma} \delta\left(\sigma, \sigma^{\prime}\right)+\partial_{\sigma^{\prime}} \delta\left(\sigma^{\prime}, \sigma\right)\right], \\
& \left(\Phi^{k}(\sigma), \Psi^{l}\left(\sigma^{\prime}\right)\right)=M^{k l} \partial_{\sigma} \partial_{\sigma^{\prime}} \delta\left(\sigma, \sigma^{\prime}\right)+m^{2}\left(B^{2}\right)^{k l} \delta\left(\sigma, \sigma^{\prime}\right), \\
& \left(\Psi^{k}(\sigma), \Psi^{l}\left(\sigma^{\prime}\right)\right)=\frac{m^{2}}{2 \pi \alpha^{\prime}} B^{k l}\left[\partial_{\sigma} \delta\left(\sigma, \sigma^{\prime}\right)+\partial_{\sigma^{\prime}} \delta\left(\sigma^{\prime}, \sigma\right)\right]
\end{aligned}
$$

and in general

$$
C^{(\alpha k \mathrm{n})(\beta l \mathrm{~m})}\left(\sigma, \sigma^{\prime}\right)=\left(\phi^{(\alpha k \mathrm{n})}(\sigma), \phi^{(\beta l \mathrm{~m})}\left(\sigma^{\prime}\right)\right)=\partial_{\sigma}^{2 \mathrm{n}} \partial_{\sigma^{\prime}}^{2 \mathrm{~m}} C^{(\alpha k 0)(\beta l 0)}\left(\sigma, \sigma^{\prime}\right) .
$$

The Dirac bracket is given by

$$
\begin{aligned}
\left(A(\sigma), B\left(\sigma^{\prime}\right)\right)^{*}= & \left(A(\sigma), B\left(\sigma^{\prime}\right)\right) \\
& -\sum_{\sigma^{\prime \prime} \sigma^{\prime \prime \prime}}\left(A(\sigma), \phi^{(\alpha k \mathrm{n})}\left(\sigma^{\prime \prime}\right)\right) C_{(\alpha k \mathrm{n})(\beta l \mathrm{~m})}\left(\sigma^{\prime \prime}, \sigma^{\prime \prime \prime}\right) \\
& \times\left(\phi^{(\beta l \mathrm{~m})}\left(\sigma^{\prime \prime \prime}\right), B\left(\sigma^{\prime}\right)\right),
\end{aligned}
$$

where the sum $\sigma^{\prime \prime}, \sigma^{\prime \prime \prime}$ is over the endpoints $0, \pi$. As in [26], the Dirac bracket can be computed similarly without knowing the explicit form of the inverse matrix $C_{(\alpha k \mathrm{n})(\beta l \mathrm{~m})}$. Only its defining properties are needed. We find the results (3.45)-(3.47). In particular, we note that the $\sigma, \sigma^{\prime}$ dependent part of the right-hand side of (3.54) and (3.56) are identical and this accounts for the fact that the commutators (3.45) and (3.46) are both given by the same function $f\left(\sigma+\sigma^{\prime}\right)$. 


\section{Scaling limits}

In this section, we will consider scaling limits of $\alpha^{\prime}, B, m$ such that the commutation relations (3.27)-(3.29) remain nontrivial. In these limits the noncommutative algebra should play a role in the D-brane physics. We remark that one can equivalently perform the limits on (3.38) for the variables $a_{0}^{i}$. Here we choose to consider the variables $x_{0}^{i}, p_{0}^{i}$ since we would like to explore and develop possible geometrical interpretations.

\subsection{Small B-field limit}

The DBI action is exact to all orders in $\alpha^{\prime}$ [38] for the $U(1)$ gauge field $\mathcal{F}$. Here we prefer to think of it as a consequence of supersymmetry (see, for example, [27-29]). For small $B$-field, it should be possible to check that the DBI action on pp-wave background is equivalent to the DBI action on the noncommutative space without $B$-field for slowly varying fields, in a similar way as it was checked for the flat background [25]. This match may provide some hint for the appropriate description of the noncommutative space. A particularly interesting limit is to go to the infinite momentum frame and take a small $B$-field simultaneously:

$$
B \sim \epsilon^{2}, \quad m \sim \frac{1}{\epsilon}, \quad g_{i j}, \quad \alpha^{\prime} \text { fixed. }
$$

The commutation relations (3.27)-(3.29) become

$$
\begin{aligned}
& {\left[x_{0}^{i}, p_{0}^{j}\right]=i g^{i j},} \\
& {\left[x_{0}^{i}, x_{0}^{j}\right]=i 2 \pi \alpha^{\prime} B^{i j},} \\
& {\left[p_{0}^{i}, p_{0}^{j}\right]=i \frac{\pi m^{2}}{2 \alpha^{\prime}} B^{i j} .}
\end{aligned}
$$

In this limit the coordinates noncommutativity can be treated perturbatively, but the description of the momentum noncommutativity has to be exact. This limit is interesting since the $p^{+}$dependence has essentially disappeared because one can treat all the strings to have the same lightcone momentum in the leading order approximation. It would be interesting to study the corresponding large $N$ matrix model.

\subsection{Large B-field limit: deformed phase space}

When $m=0$, all the excited modes can be ignored in the limit $\alpha^{\prime} \rightarrow 0$. And we only need to consider the zero modes for the low energy effective theory. Moreover, if we also take the large $B$-field limit [25] of Seiberg and Witten:

$$
\alpha^{\prime} \sim \epsilon^{1 / 2}, \quad g_{i j} \sim \epsilon, \quad \bar{B} \sim \epsilon^{-1 / 2},
$$

then a noncommutative theory is obtained with $M$ (3.9) and the noncommutativity parameter

$$
\theta^{i j}:=2 \pi \alpha^{\prime}\left(g^{-1} B M^{-1}\right)^{i j}=\frac{2 \pi b^{i j}}{b^{2}}
$$


fixed and finite in the limit. To facilitate the comparison, our $B_{i j}$ is related to the dimensionful $b_{i j}$ of Seiberg-Witten [25] by $B_{i j}=\alpha^{\prime} b_{i j}$. Hence $\bar{B}=\alpha^{\prime} b / \lambda \sim \epsilon^{-1 / 2}$ as $b$ is fixed.

However, for $m \neq 0$, the zero mode frequency $\omega_{0}$ is of the same order of magnitude as the oscillator modes, and the ratio $\omega_{0} / \omega_{1}$ is finite in the limit $\alpha^{\prime} \rightarrow 0$. Therefore, generally we cannot ignore the higher frequency modes unless we take another suitable limit at the same time. It is easy to see that for fixed $m$, the Seiberg-Witten limit (4.5) does the job. It sends $\omega_{0} \sim \epsilon^{1 / 2}$ while keeping $\omega_{n \neq 0}$ fixed. The zero modes commutation relations take the form

$$
\begin{aligned}
& {\left[x_{0}^{i}, p_{0}^{j}\right]=i h^{i j},} \\
& {\left[x_{0}^{i}, x_{0}^{j}\right]=i \theta^{i j},} \\
& {\left[p_{0}^{i}, p_{0}^{j}\right]=i \kappa^{i j},}
\end{aligned}
$$

where $\theta^{i j}$ is given by (4.6) and

$$
h^{i j}:=\frac{\lambda}{\alpha^{\prime 2} b^{2}} \frac{\pi m}{\tanh \pi m} \delta^{i j}, \quad \kappa^{i j}:=\frac{\lambda^{2} m^{2}}{4 \alpha^{\prime 4} b^{2}} \theta^{i j}
$$

are fixed and finite in the limit. Note that $\theta$ has dimension (length) ${ }^{2}$ and $\kappa$ has dimension (length) $)^{-2}$. Note also that the reason that $\kappa \propto \theta$ is because here we are dealing with 2 dimensions only. This will not be the case if we consider a higher rank $B$-field.

The commutation relations (4.7)-(4.9) are intriguing. They represent a phase space with a fully noncommutative structure. If $m$ is zero, we go back to the usual case of a theory defined on a noncommutative manifold. For $m \neq 0$, a deformed phase space emerges with the noncommutativity parameters $\theta^{i j}$ and $\kappa^{i j}$. Moreover, these parameters depends on the pp-wave background and the string momentum. It will be very interesting to understand the implications of this.

As a simpler question, one can momentarily forget about the string embedding and consider simply the physics of the noncommutative phase space by itself. Since the phase space is fully noncommutative in terms of these variables $x_{0}, p_{0}$, the usual formulation of quantum field theory using a momentum representation will have to be examined. We will call a phase space which satisfies the relations (4.7)-(4.9) (not necessarily with $\kappa \propto \theta$ ) a deformed phase space. It will be very interesting to try to construct a field theory which represents the structure of this deformed phase space.

\section{Discussions}

In this paper, we presented the quantization of the open string ending on a D-brane in pp-wave background with a constant $B$-field. We found that, due to the combined effects of the $B$-field and the mass parameter, the noncommutative structure of the theory takes on a new form. Compared with the case of a noncommutative D-brane in a flat background, the novelty of the noncommutative algebra we obtained in this paper is the noncommutativity of the linear momentum: (3.46) at the level of the string coordinates, and (3.29) at the level of the zero modes. And moreover, the boundary commutation relations depend on 
the lightcone momentum $p^{+}$of the string. In fact, the momentum is also noncommutative for charged open strings, and similarly, for open strings with their endpoints ending on different $\mathrm{D}$-branes with different background fields $\mathcal{F}$. Since no state can be a simultaneous eigenstate for all components of the momentum, it is unclear whether one can use the usual Fourier analysis to define the $*$-product as before. (See [39] for a proposal for the case of open strings ending on D-branes with different backgrounds.) Furthermore, in all of these cases, the linear momentum is not a conserved quantity. Rather, the angular momentum is conserved. It would be very interesting if this leads to a new class of noncommutative field theories and to investigate their properties.

As in the neutral case, the novelty of the noncommutativity resides in the zero modes sector. In the neutral string case, the effect of noncommutativity of the zero modes in the operator formalism was noticed in [34]. This led directly to the construction of the general multiloop amplitudes for a noncommutative open string using the Reggeon formalism [40]. In the charged string case, the noncommutativity of the zero modes played an important role in the determination of the charged string spectrum [35] and the partition function [36]. Moreover, the charged string propagator has been constructed [41] which utilized essentially the noncommutativity of the zero modes. Aspects in the understanding of the charged string interaction were recently made in [42]. In our present case, we have determined some immediate physical effects of the commutation relations we obtained in this paper. In terms of the oscillator algebra, (3.34), (3.36) or (3.38), it implies a splitting in the zero mode spectrum. It also implies a more complicated mode number dependence of the nonzero mode spectrum. Written in terms of the phase space variables, the zero modes noncommutativity takes the form (3.27)-(3.29) generically depending on the lightcone momentum of the string and is thus, in some sense, probe dependent. This is an interesting phenomena. A probe dependent phase space may sound strange. However, if geometry can be probe dependent (see, for example, [43] for an introduction to various aspects of quantum geometry), it may not be unnatural to expect an extension to that of a probe dependent phase space. This is a fundamental problem. We have not understood its significance. However, it is an intriguing possibility. Although our results were derived in a background without gravity, it is conceivable that quantum gravity at the Planck scale may also involve a similar problem, and therefore lessons that can be drawn from this construction should be helpful.

Naively, the commutation relations of the deformed phase space lead to the phase space uncertainty relations

$$
\Delta x^{i} \Delta p^{j} \sim\left|h^{i j}\right|, \quad \Delta x^{i} \Delta x^{j} \sim\left|\theta^{i j}\right|, \quad \Delta p^{i} \Delta p^{j} \sim\left|\kappa^{i j}\right| .
$$

Along the lines of [44], this might be used to generalize the spacetime uncertainty relations of Yoneya (see [45] for a review and further references). It would be interesting to see how string dualities may put constraints (e.g., string coupling dependence [44]) on the forms of these phase space uncertainty relations. We remark that (5.1) does not fall into the classification of quantum spacetime of [46]. It would be interesting to investigate what kinds of assumptions on the quantum phase space could lead to the above uncertainty relations.

Due to the $p^{+}$-dependence in some of our commutation relations, it is not clear whether one can apply the Mandelstam lightcone formalism. It is important to understand this issue. 
Assuming we can overcome the problem discussed above and have gained sufficient control over string scattering in the GS formulation, it will also be very interesting to understand the results obtained in this paper, particularly the momentum noncommutativity, in the approach of Schomerus [47]. This will be useful for the understanding of the properties of the D-brane low energy theory.

\section{Acknowledgements}

C.S.C. thanks Simon Ross for helpful discussions and Rodolfo Russo for useful comments. The work of C.S.C. was supported in part by the Nuffield Foundation, Grant Number NUF-NAL/00445/G. The work of P.M.H. is supported in part by the National Science Council, the Center for Theoretical Physics at National Taiwan University, the National Center for Theoretical Sciences, and the CosPA project of the Ministry of Education, Taiwan, ROC.

\section{References}

[1] M. Blau, J. Figueroa-O’Farrill, C. Hull, G. Papadopoulos, A new maximally supersymmetric background of IIB superstring theory, JHEP 0201 (2002) 047, hep-th/0110242.

[2] M. Blau, J. Figueroa-O'Farrill, C. Hull, G. Papadopoulos, Penrose limits and maximal supersymmetry, hepth/0201081.

[3] M. Blau, J. Figueroa-O’Farrill, G. Papadopoulos, Penrose limits, supergravity and brane dynamics, hepth/0202111.

[4] R. Penrose, Any spacetime has a plane wave limit, in: Differential Geometry and Relativity, Riedel, Dordrecht, 1976.

[5] R.R. Metsaev, Type IIB Green-Schwarz superstring in plane wave Ramond-Ramond background, Nucl. Phys. B 625 (2002) 70, hep-th/0112044.

[6] R.R. Metsaev, A.A. Tseytlin, Exactly solvable model of superstring in plane wave Ramond-Ramond background, hep-th/0202109.

[7] J.G. Russo, A.A. Tseytlin, On solvable models of type IIB superstring in NS-NS and R-R plane wave backgrounds, hep-th/0202179.

[8] M. Billo, I. Pesando, Boundary states for GS superstrings in an Hpp wave background, hep-th/0203028.

[9] J. Maldacena, The large $N$ limit of superconformal field theories and supergravity, Adv. Theor. Math. Phys. 2 (1998) 231, hep-th/9711200.

[10] D. Berenstein, J. Maldacena, H. Nastase, Strings in flat space and pp waves from $N=4$ super-Yang-Mills, hep-th/0202021.

[11] N. Itzhaki, I.R. Klebanov, S. Mukhi, PP wave limit and enhanced supersymmetry in gauge theories, hepth/0202153.

[12] J. Gomis, H. Ooguri, Penrose limit of $N=1$ gauge theories, hep-th/0202157.

[13] L.A. Zayas, J. Sonnenschein, On Penrose limits and gauge theories, hep-th/0202186.

[14] M. Alishahiha, M.M. Sheikh-Jabbari, The $p p$-wave limits of orbifolded $A d S(5) \times S * * 5$, hep-th/0203018.

[15] N. Kim, A. Pankiewicz, S.J. Rey, S. Theisen, Superstring on pp-wave orbifold from large- $N$ quiver gauge theory, hep-th/0203080.

[16] M. Cvetič, H. Lu, C.N. Pope, Penrose limits, pp-waves and deformed M2-branes, hep-th/0203082.

[17] T. Takayanagi, S. Terashima, Strings on orbifolded pp-waves, hep-th/0203093.

[18] U. Gursoy, C. Nunez, M. Schvellinger, RG flows from Spin(7), CY 4-fold and HK manifolds to AdS, Penrose limits and pp waves, hep-th/0203124.

[19] E. Floratos, A. Kehagias, Penrose limits of orbifolds and orientifolds, hep-th/0203134. 
[20] R. Gueven, Randall-Sundrum zero mode as a Penrose limit, hep-th/0203153.

[21] S.R. Das, C. Gomez, S.-J. Rey, Penrose limit, spontaneous symmetry breaking and holography in pp-wave background, hep-th/0203164.

[22] A. Connes, M.R. Douglas, A. Schwarz, Noncommutative geometry and matrix theory: compactification on tori, JHEP 9802 (1998) 003, hep-th/9711162.

[23] M.R. Douglas, C.M. Hull, D-branes and the noncommutative torus, JHEP 9802 (1998) 008, hep-th/9711165.

[24] C.S. Chu, P.M. Ho, Noncommutative open string and D-brane, Nucl. Phys. B 550 (1999) 151, hepth/9812219.

[25] N. Seiberg, E. Witten, String theory and noncommutative geometry, JHEP 9909 (1999) 032, hep-th/9908142.

[26] C.S. Chu, P.M. Ho, Constrained quantization of open string in background $B$-field and noncommutative D-brane, Nucl. Phys. B 568 (2000) 447, hep-th/9906192.

[27] M. Aganagic, C. Popescu, J.H. Schwarz, D-brane actions with local kappa symmetry, Phys. Lett. B 393 (1997) 311, hep-th/9610249;

M. Aganagic, C. Popescu, J.H. Schwarz, Gauge-invariant and gauge-fixed D-brane actions, Nucl. Phys. B 495 (1997) 99, hep-th/9612080.

[28] M. Cederwall, A. von Gussich, B.E. Nilsson, P. Sundell, A. Westerberg, The Dirichlet super-p-branes in ten-dimensional type IIA and IIB supergravity, Nucl. Phys. B 490 (1997) 179, hep-th/9611159.

[29] E. Bergshoeff, P.K. Townsend, Super D-branes, Nucl. Phys. B 490 (1997) 145, hep-th/9611173.

[30] E. Bergshoeff, R. Kallosh, T. Ortin, G. Papadopoulos, Kappa-symmetry, supersymmetry and intersecting branes, Nucl. Phys. B 502 (1997) 149, hep-th/9705040.

[31] M. Berkooz, M.R. Douglas, R.G. Leigh, Branes intersecting at angles, Nucl. Phys. B 480 (1996) 265, hepth/9606139.

[32] A. Bilal, C.S. Chu, D3 brane(s) in $A d S(5) \times S(5)$ and $N=4,2,1$ SYM, Nucl. Phys. B 547 (1999) 179, hep-th/9810195.

[33] M.B. Green, M. Gutperle, Light-cone supersymmetry and D-branes, Nucl. Phys. B 476 (1996) 484, hepth/9604091.

[34] C.S. Chu, F. Zamora, Manifest supersymmetry in non-commutative geometry, JHEP 0002 (2000) 022, hepth/9912153.

[35] A. Abouelsaood, C.G. Callan, C.R. Nappi, S.A. Yost, Open strings in background gauge fields, Nucl. Phys. B 280 (1987) 599.

[36] C. Bachas, M. Porrati, Pair creation of open strings in an electric field, Phys. Lett. B 296 (1992) 77, hepth/9209032.

[37] C.S. Chu, Noncommutative open string: neutral and charged, hep-th/0001144.

[38] E.S. Fradkin, A.A. Tseytlin, Nonlinear electrodynamics from quantized strings, Phys. Lett. B 163 (1985) 123.

[39] K. Dasgupta, Z. Yin, Non-Abelian geometry, hep-th/0011034.

[40] C.S. Chu, R. Russo, S. Sciuto, Multiloop string amplitudes with $B$-field and noncommutative QFT, Nucl. Phys. B 585 (2000) 193, hep-th/0004183.

[41] L. Dolan, C.R. Nappi, A scaling limit with many noncommutativity parameters, Phys. Lett. B 504 (2001) 329, hep-th/0009225.

[42] C.S. Chu, R. Russo, S. Sciuto, Remarks on the calculations of charged open string amplitudes: the 1-loop tadpole, hep-th/0201118.

[43] B.R. Greene, Lectures on quantum geometry, Nucl. Phys. (Proc. Suppl.) 41 (1995) 92.

[44] C.S. Chu, P.M. Ho, Y.C. Kao, Worldvolume uncertainty relations for D-branes, Phys. Rev. D 60 (1999) 126003 , hep-th/9904133.

[45] T. Yoneya, String theory and space-time uncertainty principle, Prog. Theor. Phys. 103 (2000) 1081, hepth/0004074.

[46] S. Doplicher, K. Fredenhagen, J.E. Roberts, The Quantum structure of space-time at the Planck scale and quantum fields, Commun. Math. Phys. 172 (1995) 187.

[47] V. Schomerus, D-branes and deformation quantization, JHEP 9906 (1999) 030, hep-th/9903205. 